\title{
Minerva: New nodules on screening lung computed tomography
}

In this story (BMJ 2016;353:i3660, doi:10.1136/bmj.i3660) Minerva said that $49 \%$ of new solid nodules detected in the
NELSON trial were cancers. In fact, a new solid nodule was lung cancer in $49(6 \%)$ participants. 